\title{
Construção de um protótipo de aplicativo móvel para processo de enfermagem do
}

\section{paciente renal}

\author{
Construction of a mobile application prototype for the renal patient's nursing process \\ Construcción de un prototipo de aplicación móvil para el proceso de enfermería del paciente renal
}

Recebido: 23/02/2021 | Revisado: 02/03/2021 | Aceito: 04/03/2021 | Publicado: 14/03/2021

\author{
Marcela Claudia de Paula Oliveira \\ ORCID: https://orcid.org/0000-0002-7762-0684 \\ Universidade Federal de Pernambuco, Brasil \\ E-mail: marcela-claudia2011@hotmail.com \\ Aline Kelly de Moura \\ ORCID: https://orcid.org/0000-0002-0649-3323 \\ Universidade Federal de Pernambuco, Brasil \\ E-mail: alinek_moura@hotmail.com \\ Kelita Mirelle Oliveira Lima \\ ORCID: https://orcid.org/0000-0002-3783-2201 \\ Universidade Federal de Pernambuco, Brasil \\ E-mail: kelita_mirelle_sp@hotmail.com \\ Maria Carolina Wanderley Costa de Medeiros \\ ORCID: https://orcid.org/0000-0001-5858-6532 \\ Universidade Federal de Pernambuco, Brasil \\ E-mail: carolwcm@gmail.com \\ Marta Nunes Lira \\ ORCID: https://orcid.org/0000-0003-3780-4187 \\ Universidade Federal de Pernambuco, Brasil \\ E-mail: martanuneslira@yahoo.com.br \\ Juliana Romano de Lima \\ ORCID: https://orcid.org/0000-0002-7404-6541 \\ Universidade Federal de Pernambuco, Brasil \\ E-mail: juliana_romano144@hotmail.com
}

\begin{abstract}
Resumo
A utilização de sistemas de tecnologia voltados para a área da saúde tem se mostrado uma excelente ferramenta no aprimoramento do cuidado prestado ao paciente/cliente, pois permite ao profissional coletar, registrar dados e se comunicar com a equipe multiprofissional de maneira mais rápida e eficaz. Trata-se de um estudo metodológico do tipo descritivo, com o objetivo de apresentar o desenvolvimento de um protótipo de aplicativo móvel de média fidelidade para o Processo de Enfermagem do paciente renal. O protótipo é composto por um total de 29 telas e foi construído com o objetivo de oferecer a um serviço hospitalar a aplicação do Processo de Enfermagem de forma rápida, precisa e segura. De maneira geral a construção das telas foi dividida em categorias: tela inicial, menu login, cadastro hospital, menu hospital, cadastro colaborador, login colaborador, menu pacientes, cadastro de paciente, opções do processo, histórico de enfermagem, exame físico, diagnósticos sugeridos, diagnósticos selecionados, plano assistencial, resultados esperados, implementação, avaliação, intercorrências, destino do paciente e registros anteriores. A partir deste estudo, tornou-se factível, a construção de um protótipo de aplicativo móvel para realização do processo de enfermagem em nefrologia e este, servirá de instrumento para a elaboração de estudos posteriores, realizados os devidos ajustes para o alcance do resultado desejado.
\end{abstract}

Palavras-chave: Tecnologia; Sistematização da assistência de enfermagem; Aplicativos móveis.

\begin{abstract}
The use of technology systems at the health area has been an excellent tool in improving the care assistance to the patient/client, as it allows the professional to collect, record data and communicate with the multiprofessional team more quickly and effectively. This is a descriptive type methodological study, with the objective of showing the development of a prototype of a mobile application of medium fidelity for the Nursing Process of the renal patient. The prototype is composed by 29 screens and was built with the intention of offering a hospital service the application of the Nursing Process quickly, accurately and safely. In general, the construction of the screens was divided into categories: home screen, login menu, hospital registration, hospital menu, collaborative registration, collaborative login, patient menu, patient registration, process options, nursing history, physical examination, suggested diagnoses, selected diagnoses, care plan, expected results, implementation, evaluation, complications, patient's destination and previous records. From this study, it became feasible to build a mobile application prototype to perform the nursing
\end{abstract}


process focused on the chronic kidney patient, this prototype will serve as an instrument for further studies, with the necessary adjustments to achieve the desired result.

Keywords: Technology; Systematization of nursing care; Mobile apps.

\section{Resumen}

El uso de sistemas tecnológicos orientados al área de la salud ha demostrado ser una excelente herramienta para mejorar la atención brindada al paciente / cliente, ya que permite al profesional recolectar, registrar datos y comunicarse con el equipo multiprofesional de manera más rápida y efectiva. Se trata de un estudio metodológico de tipo descriptivo, con el objetivo de presentar el desarrollo de un prototipo de una aplicación móvil de mediana fidelidad para el Proceso de Enfermería del paciente renal. El prototipo consta de un total de 29 pantallas y fue construido con el objetivo de ofrecer un servicio hospitalario la aplicación del Proceso de Enfermería de forma rápida, precisa y segura. En general, la construcción de las pantallas se dividió en categorías: pantalla de inicio, menú de inicio de sesión, registro del hospital, menú del hospital, registro colaborativo, inicio de sesión colaborativo, menú del paciente, registro del paciente, opciones de proceso, historial de enfermería, examen físico, diagnósticos sugeridos, seleccionados diagnósticos, plan de cuidados, resultados esperados, implementación, evaluación, complicaciones, destino del paciente y registros previos. A partir de este estudio se hizo factible la construcción de un prototipo de aplicación móvil para llevar a cabo el proceso de enfermería en nefrología y este servirá de instrumento para la elaboración de estudios posteriores, con los ajustes necesarios para lograr el resultado deseado.

Palabras clave: Tecnología; Sistematización de la atención de enfermería; Aplicaciones móviles.

\section{Introdução}

O século XX marca o início da era do conhecimento e da informação, caracterizada por um período de grandes mudanças no cenário tecnológico, social e econômico. A utilização das Tecnologias de Informação (TI) tornou-se parte do cotidiano da população mundial, considerada uma necessidade básica, popular e disponível nos dispositivos móveis. O uso das tecnologias móveis trouxe mudanças nas estratégias de ensino-aprendizagem teórico e prático. No ano de 2013 foram baixados mais de 100 bilhões de aplicativos, esses números ultrapassaram a marca dos 200 bilhões apenas em 2016. (Oliveira, 2017; Tibes, 2015).

Dentre as tecnologias para a saúde, o registro eletrônico permite acesso imediato às informações, melhora a eficiência do processo de trabalho, acessibilidade e velocidade na comunicação entre a equipe multiprofissional. A evolução tecnológica trouxe consigo a possibilidade de construir o conhecimento, disseminar informações e dessa maneira revolucionar o desenvolvimento científico e tecnológico em várias áreas dos modelos de trabalho, inclusive da enfermagem. Tais avanços se expressaram com a introdução da informática e o aparecimento de aparelhos modernos e sofisticados, trazendo benefícios e rapidez no diagnóstico e tratamento das doenças (Santos Barros, 2019; Pissaia et al. 2017).

A inovação tecnológica facilita a atividade profissional na área da saúde favorecendo a coleta de dados e registros de informações sobre o paciente e o estado de adoecimento reduzindo o tempo gasto na documentação das atividades realizadas e diminuindo o risco de perda das informações, uma vez que, serão armazenadas em dispositivo móvel, dispensando o registro em papel físico (Rezende, Santos \& Medeiros, 2016).

Para Martins e Chianca (2016), a Sistematização da Assistência de Enfermagem (SAE) é um método que facilita o registro de dados, formaliza o cuidado personalizado e direciona com segurança e respaldo científico as atividades realizadas pela equipe de enfermagem. No Brasil, o modelo mais conhecido e seguido para a implantação do PE foi o proposto em 1979 por Wanda de Aguiar Horta, no qual propunha as seguintes fases: histórico de enfermagem, diagnóstico de enfermagem, plano assistencial, prescrição de enfermagem, avaliação de enfermagem e prognóstico de enfermagem. Na primeira etapa, serão realizadas a anamnese e exame físico através da qual será possível identificar os diagnósticos de enfermagem (DE). Na etapa seguinte, o enfermeiro deverá planejar como resolver ou minimizar os problemas identificados na fase do DE e em seguida implementar os cuidados de enfermagem. É na etapa de avaliação da assistência de enfermagem que serão monitoradas as respostas apresentadas pelos pacientes (Martins \& Chianca 2016). 
A implantação da SAE através do desenvolvimento de softwares contribui para a padronização das informações, promovendo maior agilidade na coleta de dados do paciente/cliente, armazenamento e recuperação de dados de maneira eficaz, permitindo, assim um aumento na qualidade da assistência. De Oliveira e Soares apontam a relevância da utilização de classificações na implementação do registro informatizado do processo de enfermagem, considerando a integração da taxonomia North American Nursing Diagnosis Association International (NANDA-I), a classificação das intervenções e atividades de enfermagem (NIC) e a classificação da avaliação dos resultados (NOC). A Taxonomia dos diagnósticos de enfermagem da NANDA-I é um esquema classificatório para auxiliar e organizar os conceitos a respeito (julgamentos de enfermagem ou diagnósticos de enfermagem) à prática de enfermagem. (Oliveira et al. 2012; Soares, Peres \& Oliveira 2018).

A Doença Renal Crônica é considerada um problema social e econômico em todo o mundo, associada a inúmeras comorbidades, bem como a altos gastos em saúde pública ocasionada pela alta taxa de hospitalização, causando grande impacto negativo na expectativa e qualidade de vida de seus portadores. Independentemente da doença de base, os principais desfechos em pacientes com DRC são as suas complicações (anemia, acidose metabólica, alteração do metabolismo mineral e desnutrição), decorrentes da perda funcional renal, óbito (principalmente por causas cardiovasculares) e falência funcional renal (Crestani Filho, 2013; Fassbinder, 2015).

Diante do contexto, o estudo teve como objetivo construir um protótipo de aplicativo móvel para auxiliar enfermeiros no processo de enfermagem e na elaboração dos diagnósticos de enfermagem, planejamento e avaliação voltado para o paciente renal.

Portanto, a questão norteadora de pesquisa é: Como um protótipo de aplicativo móvel possibilitará a sistematização do processo de enfermagem para o paciente renal?

\section{Metodologia}

Trata-se de um estudo qualitativo de natureza descritivo, onde os métodos qualitativos são aqueles nos quais é importante a interpretação dos dados por parte do pesquisador assim como suas opiniões sobre o fenômeno estudado. com o objetivo de apresentar o desenvolvimento de um protótipo de aplicativo móvel de média fidelidade para o Processo de Enfermagem do paciente renal (Pereira et al. 2018).

No estudo metodológico o pesquisador tem como meta a elaboração de um instrumento confiável, preciso e de fácil manipulação que possa ser utilizado por outros pesquisadores e outras pessoas (Polit \& Hungler 1995). Este tipo de estudo consiste em uma pesquisa que se refere à investigação de métodos de obtenção, organização e análise de dados, descrevendo a elaboração, validação e avaliação de ferramentas e técnicas de pesquisa (Polit \& Beck 2011).

\subsection{Desenvolvimento do protótipo}

\section{a. Etapa I}

A primeira etapa da construção do protótipo consistiu na elaboração de um instrumento para coleta de dados tendo como referencial o modelo teórico das Necessidades Humanas Básicas de Wanda Horta.

O modelo teórico de Horta possui fases inter-relacionadas e organizadas que servem para o levantamento de dados necessários para que o enfermeiro possa direcionar as intervenções para a assistência ao paciente.

Após a elaboração do instrumento de coleta de dados, teve início a realização de levantamento dos Diagnósticos de Enfermagem mais relevantes no cuidado ao paciente renal utilizando a classificação proposta pela NANDA Internacional (NANDA-I). 
Estabelecidos os diagnósticos, realizou-se o levantamento das intervenções relacionadas aos diagnósticos contidas na Nursing Interventions Classification (NIC). Estas intervenções foram utilizadas para elaboração das prescrições de enfermagem que constam no protótipo.

\section{b. Etapa II}

A segunda etapa do planejamento da construção do protótipo foi a elaboração de um fluxograma do percurso realizado pelos usuários na interface. Nesta etapa, foram estabelecidos os principais requisitos deste fluxo baseando-se em dados obtidos na revisão de literatura.

\section{c. Etapa III}

Trata-se da construção do protótipo através criação das telas por meio da plataforma FIGMA. O Figma é um editor gráfico on-line totalmente gratuito e tem como um de seus objetivos o desenvolvimento de prototipagem de interface gráfica de usuários. Este pode ser acessado através do site: www.figma.com.

Nessa etapa criou-se o cenário de telas utilizando um paciente hipotético, do qual foram gerados as interfaces de Histórico, Exame Físico, Diagnósticos, Planejamento, Implementação e Avaliação de Enfermagem.

\section{Resultados}

O protótipo foi construído com o objetivo de oferecer a um serviço hospitalar a aplicação do Processo de Enfermagem de maneira ágil, precisa e segura. Sendo assim, os enfermeiros ao utilizarem o referido sistema, podem dedicar-se as atividades assistenciais de forma mais sistemática, otimizando o tempo de suas atividades gerenciais.

O protótipo é composto por um total de 29 telas, de modo geral a construção das telas foi dividida nas seguintes categorias: tela inicial, menu login, cadastro hospital, menu hospital, cadastro colaborador, login colaborador, menu pacientes, cadastro de paciente, opções do processo, histórico de enfermagem, exame físico, diagnósticos sugeridos, diagnósticos selecionados, plano assistencial, resultados esperados, implementação, avaliação, intercorrências, destino do paciente e registros anteriores.

A primeira tela a ser exibida contém o menu inicial de boas-vindas que seguirá automaticamente para a tela menu login. Nesta tela, o colaborador do hospital acessará o sistema através do login e senha cadastrada previamente pelo fornecedor do aplicativo, onde realizará o preenchimento do cadastro com os dados do hospital e incluirá os colaboradores que irão utilizar o aplicativo no setor conforme a Figura 1. 
Figura 1 - Telas de Boas-vindas, Login e Menu do Hospital. Recife, 2020.
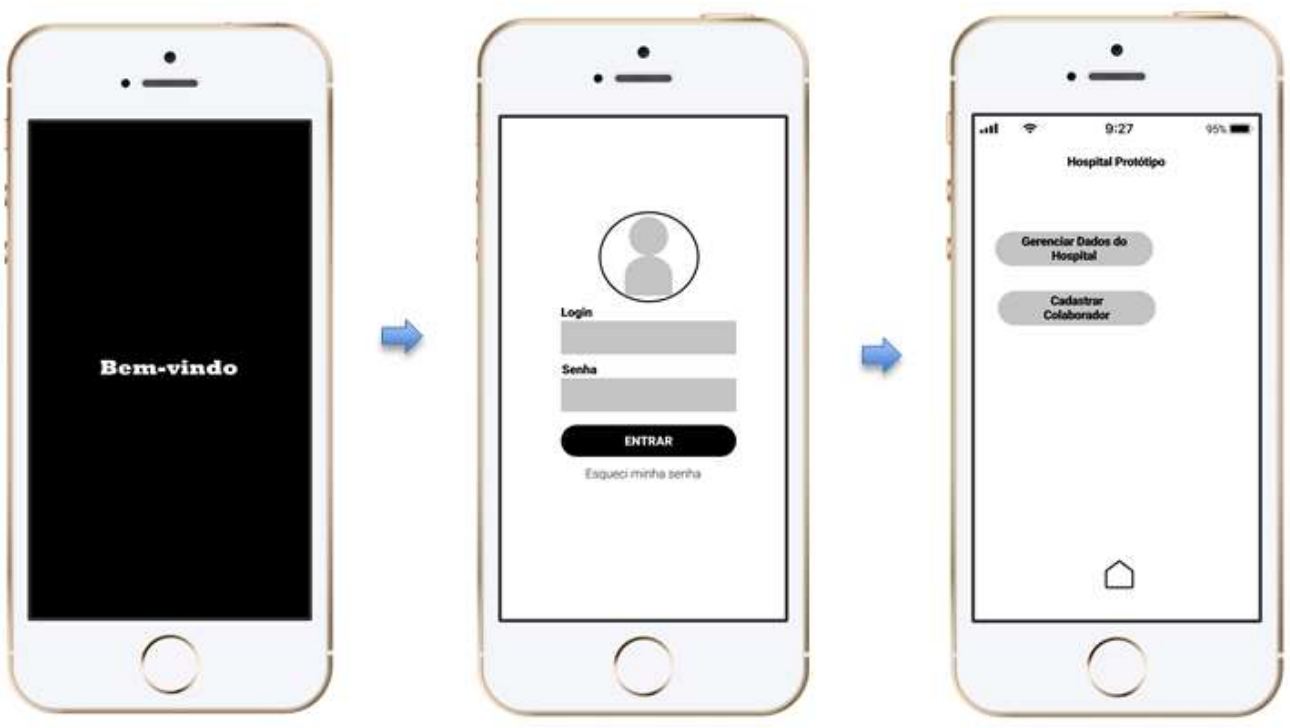

Fonte: Autores.

Figura 2 - Tela Menu Hospital e Cadastro Hospital. Recife, 2020.
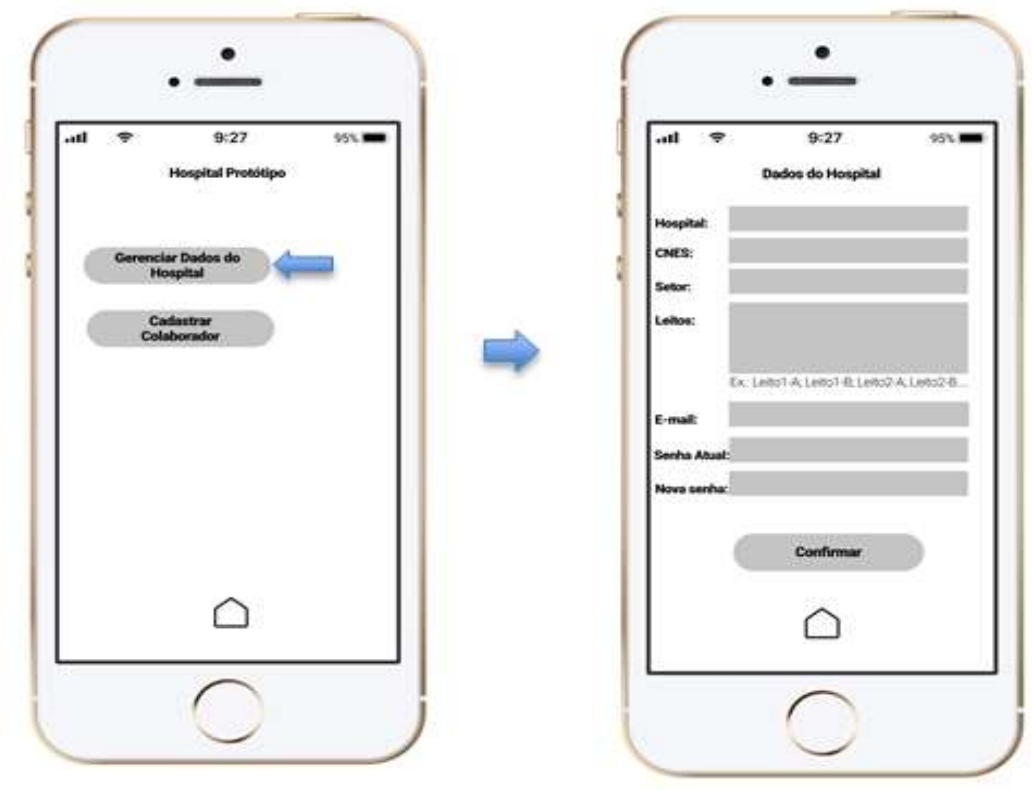

Fonte: Autores. 
Figura 3 - Tela Menu Hospital e Cadastro do Colaborador. Recife, 2020.

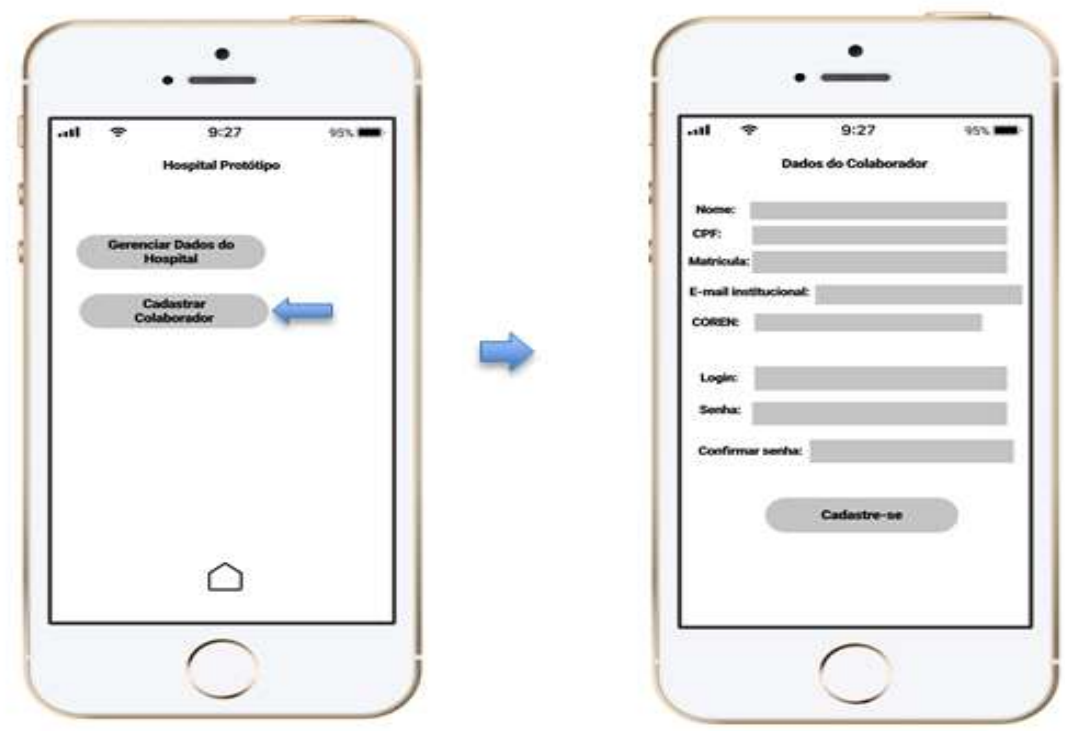

Fonte: Autores.

O usuário hospital realiza o cadastro dos colaboradores responsáveis pelo uso do aplicativo (equipe de enfermagem) permitindo assim, que o colaborador cadastrado acesse o sistema com o login e senha pessoal e seja encaminhado a tela com a lista dos leitos do setor de nefrologia do hospital. Nesta tela, o enfermeiro irá realizar o gerenciamento dos leitos: incluir e retirar pacientes, monitorar o tempo de avaliação de suas intervenções e selecionar pacientes para realizar o processo de enfermagem conforme a Figura 4.

A tela de gerenciamento de leitos (a direita na Figura 4) permite visualizar o nome e dados de todos os pacientes internos, os leitos vagos, ícone de incluir/editar dados do paciente ( ), ícone de destino do paciente ( ) e sinais de alerta coloridos. Esses sinais indicam que: o paciente teve o processo de enfermagem concluído nas últimas $24 \mathrm{~h}$ pela avaliação das intervenções de enfermagem (círculo verde), paciente com avaliação não realizada nas últimas 24h (círculo vermelho) e leito vago (círculo amarelo). 
Figura 4 - Tela de Login e Gerenciamento de Leitos. Recife, 2020.

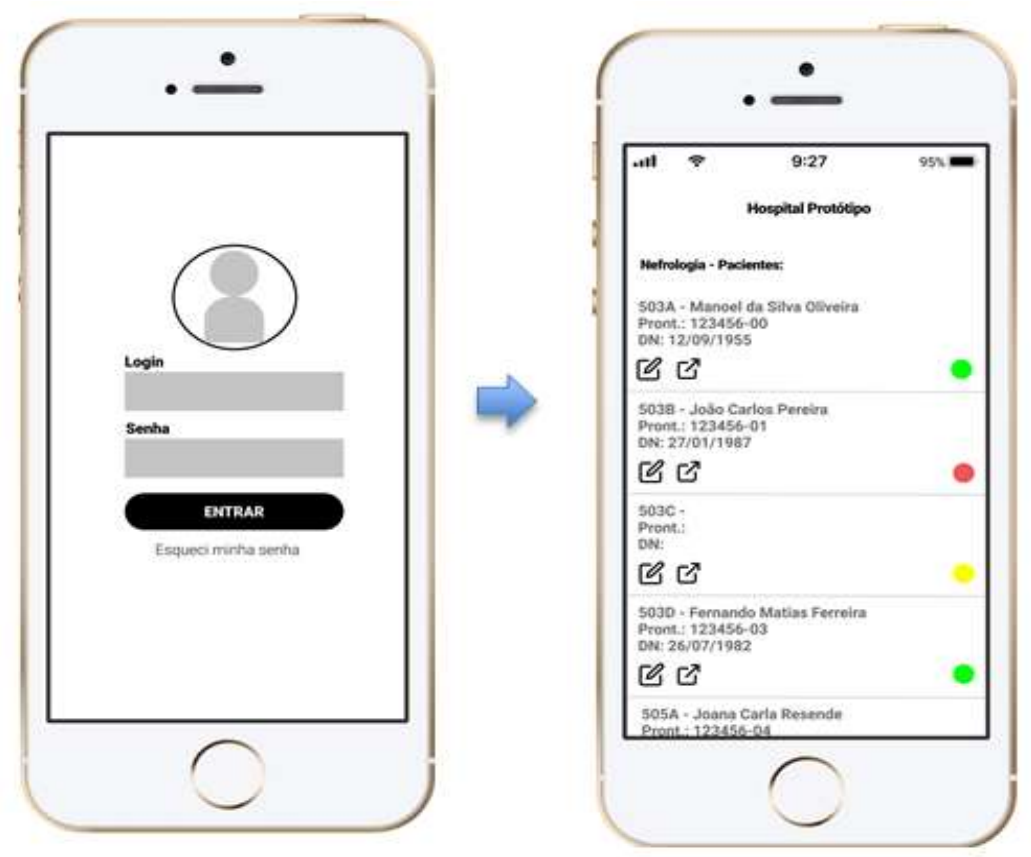

Fonte: Autores.

A Figura 5 demonstra como o enfermeiro deve proceder ao realizar a inclusão/admissão do paciente renal através do preenchimento dos dados cadastrais. Ao clicar no leito vago ou no ícone, o enfermeiro será direcionado a tela Cadastro do Paciente. Ao término do cadastro, o nome e dados do paciente aparecem na lista da tela de Gerenciamento de Leitos.

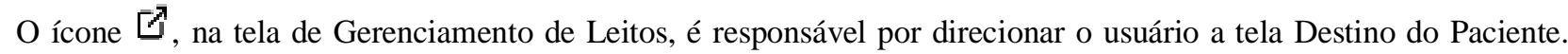
Nesta tela, é possível selecionar o destino do paciente como: alta, óbito, transferência, evasão e desistência do tratamento (Figura 6). A seleção de qualquer um dos itens através da caixa de seleção exclui automaticamente o nome do paciente da lista na tela de Gerenciamento de Leitos. 
Research, Society and Development, v. 10, n. 3, e21810313226, 2021

(CC BY 4.0) | ISSN 2525-3409 | DOI: http://dx.doi.org/10.33448/rsd-v10i3.13226

Figura 5 - Tela de Gerenciamento de Leito e Cadastro do Paciente. Recife, 2020.
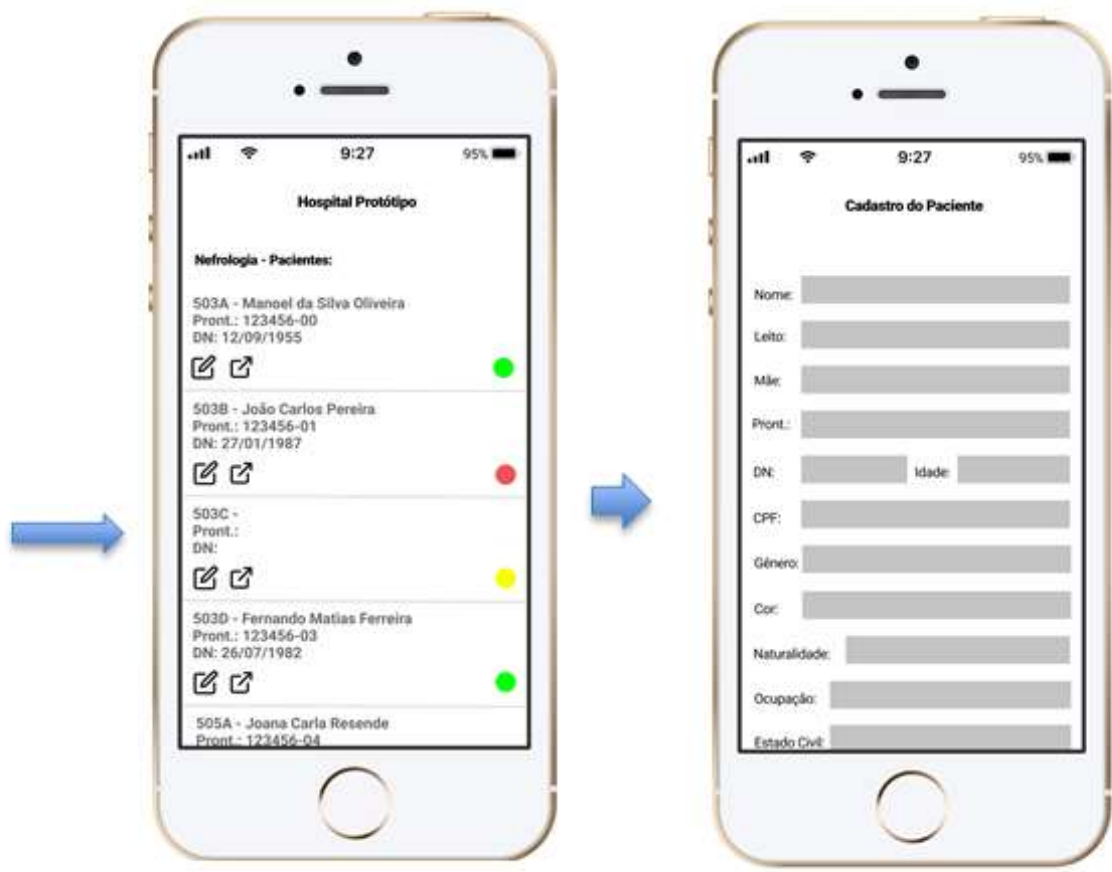

Fonte: Autores.

Figura 6 - Telas de Gerenciamento de Leitos e Destino do Paciente. Recife, 2020.
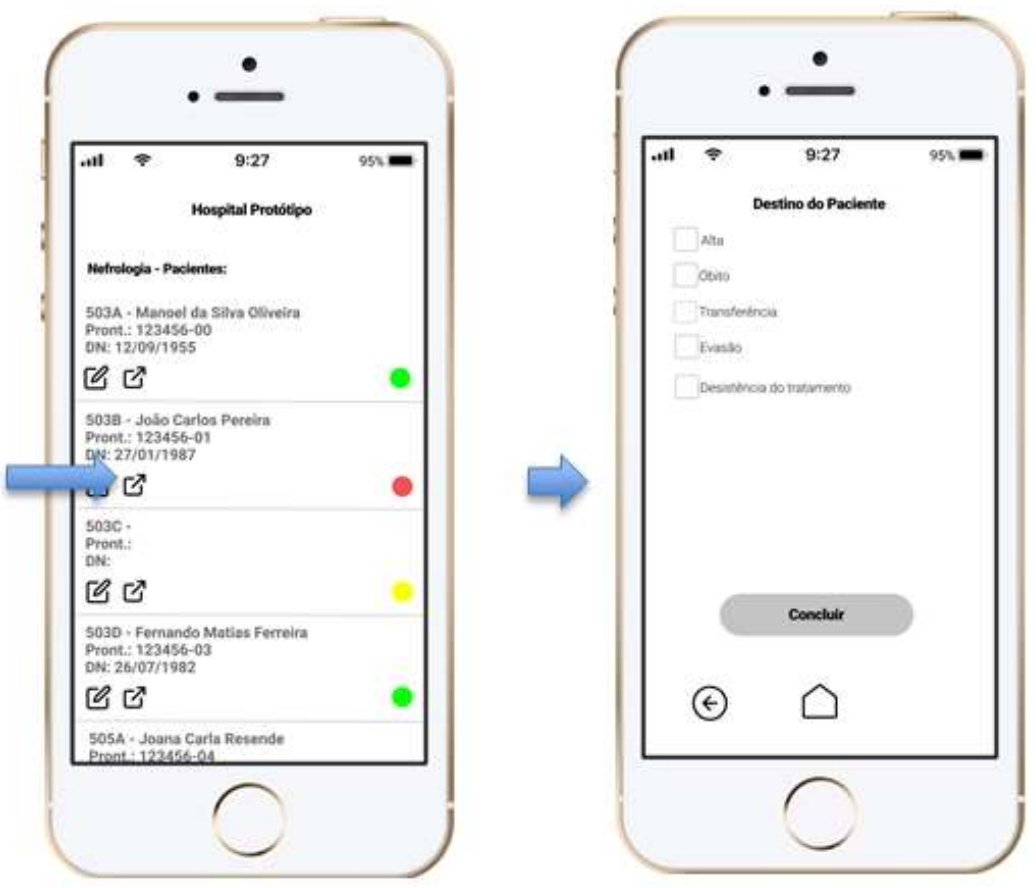

Fonte: Autores.

Como demonstrado na Figura 7, ao estar cadastrado no sistema o paciente pode ter todos os seus dados registrados e analisados seguindo o processo de enfermagem. Sendo assim, ao clicar no nome do paciente, surge para o colaborador as 
opções: Histórico de Enfermagem, Exame Físico, Planejamento e Implementação de Enfermagem, Avaliação de Enfermagem, Intercorrências e Registros Anteriores.

Figura 7 - Telas Gerenciamento de Leitos e Processo de Enfermagem. Recife, 2020.

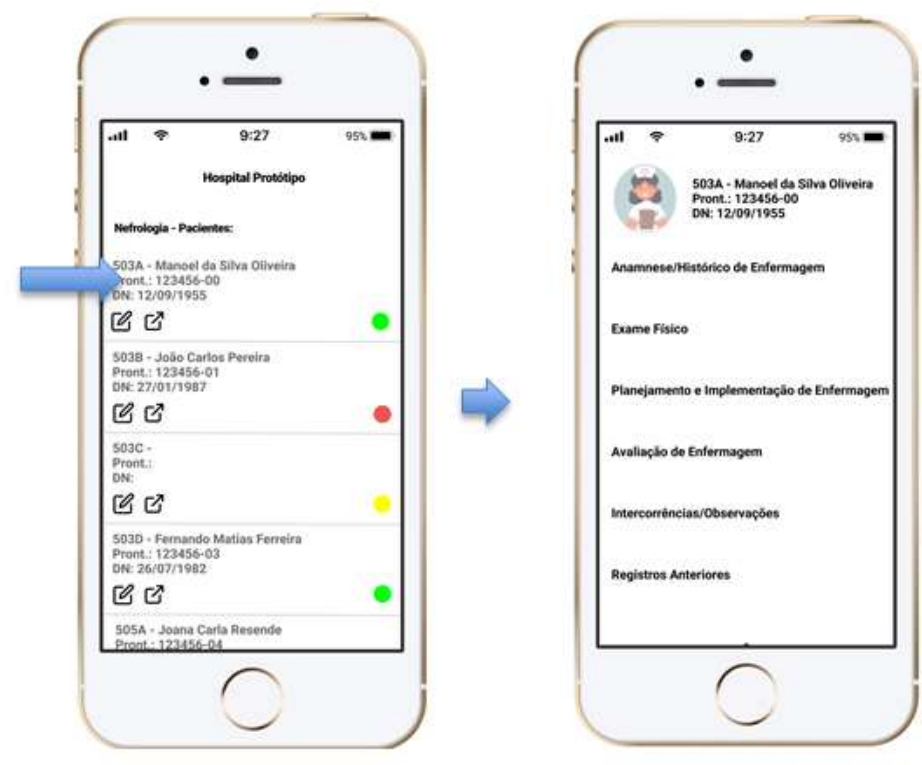

Fonte: Autores.

O Histórico de Enfermagem é composto por 4 telas, no qual o enfermeiro realiza os registros dos dados coletados de forma rápida através de caixa de seleção (checkbox) e lista suspensa (drop-down). As informações que abrangem dados subjetivos da entrevista são digitados livremente pelo usuário. Ao concluir o Histórico de Enfermagem o enfermeiro seleciona se segue para o Exame Físico ou se conclui o Histórico de Enfermagem sem o Exame Físico conforme demonstrado na Figura 8.

O Exame Físico é composto por 9 telas. A maioria dos dados registrados no exame físico são realizados através de caixa de seleção, tornando mais dinâmico e rápido o registro pelo enfermeiro. As Escalas de Glasgow, Morse e Braden contidas dentro do exame físico, tem sua pontuação e classificação calculadas automaticamente após a seleção dos itens contidos em cada escala. Ao término do Exame Físico o usuário pode apenas concluir o exame ou pode gerar os Diagnósticos de Enfermagem automaticamente (Figura 9). 
Figura 8 - Telas Histórico de Enfermagem. Recife, 2020.
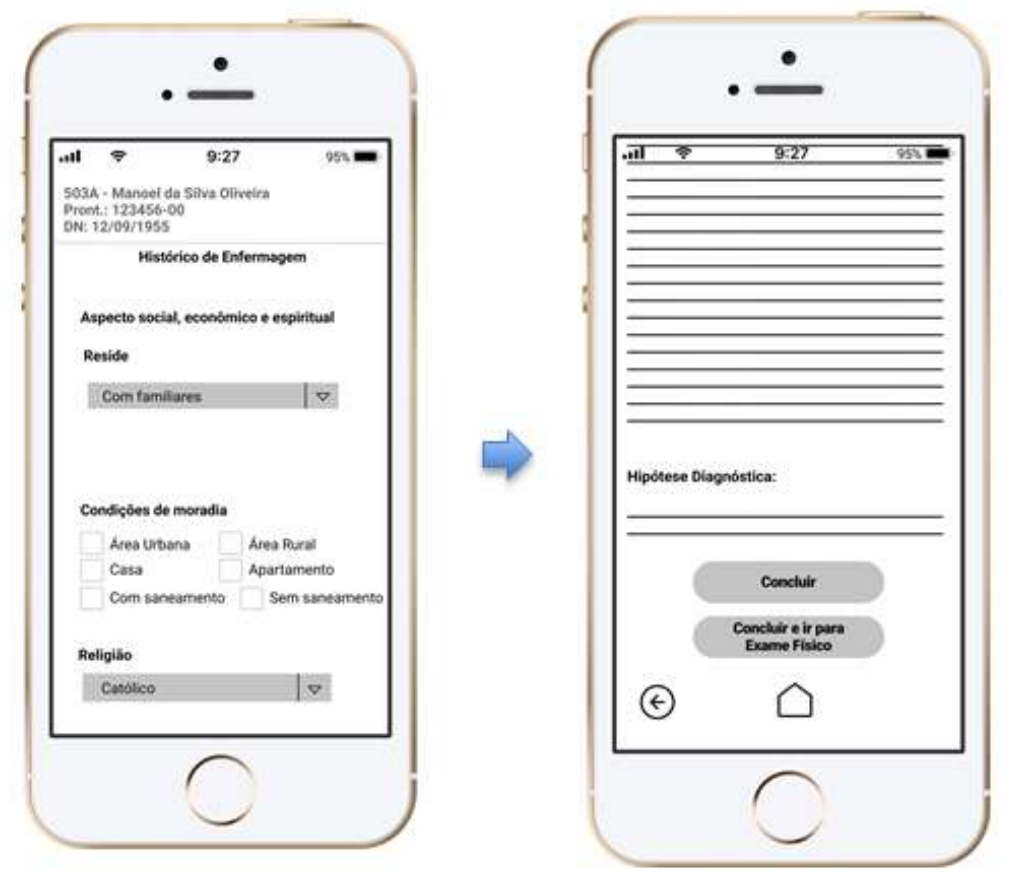

Fonte: Autores.

Figura 9 - Tela de Exame Físico. Recife, 2020.
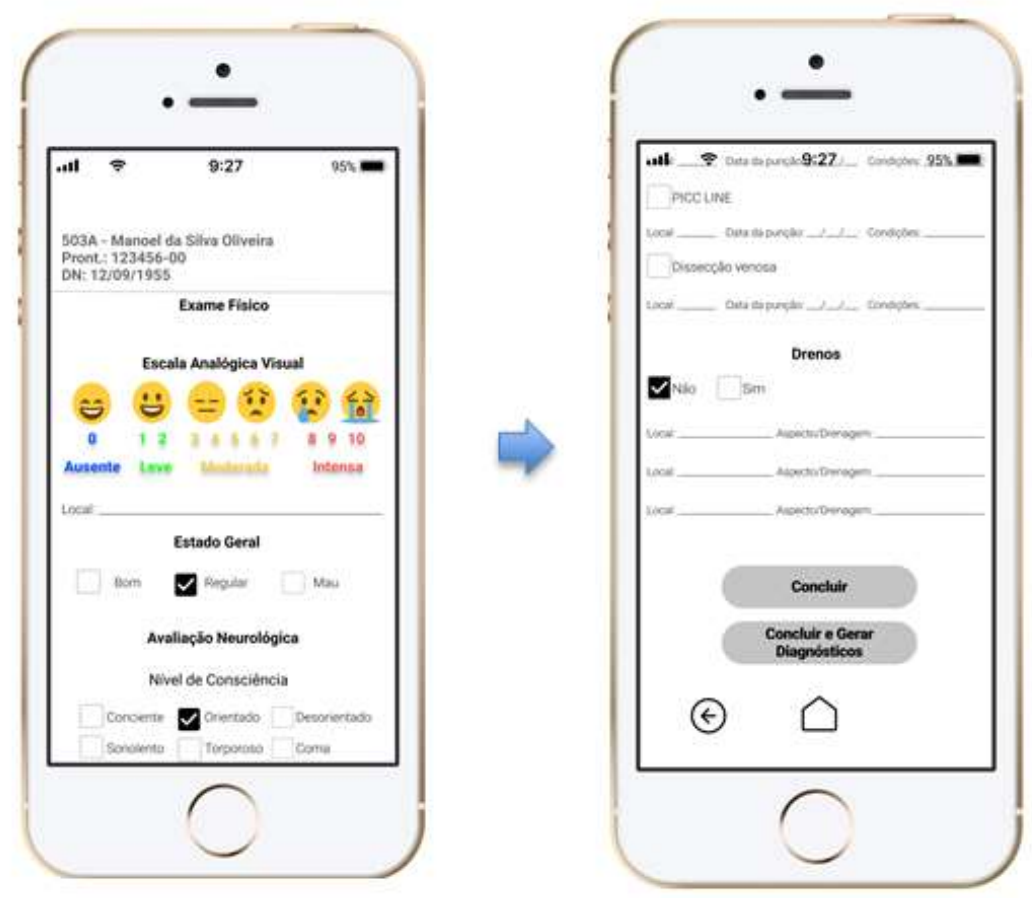

Fonte: Autores.

O protótipo desenvolvido neste trabalho tem como principal contribuição às atividades do enfermeiro e do processo de enfermagem, ser capaz de gerar os diagnósticos automaticamente a partir de características definidoras, fatores relacionados ou condições associadas registradas pelo enfermeiro durante o exame físico e histórico de enfermagem. Tornando esta ação uma ferramenta diferencial do protótipo, devido sua capacidade de contribuir com o enfermeiro no momento de executar o 
raciocínio clínico (Figura 10). A lista de Diagnósticos Sugeridos orienta o enfermeiro as melhores possibilidades para a escolha de um diagnóstico adequado a condição clínica do paciente, porém cabe ao enfermeiro selecionar os diagnósticos que julgar mais relevantes (Figura 11).

Figura 10 - Telas de finalização do Exame Físico e tela de Diagnósticos Sugeridos. Recife, 2020.
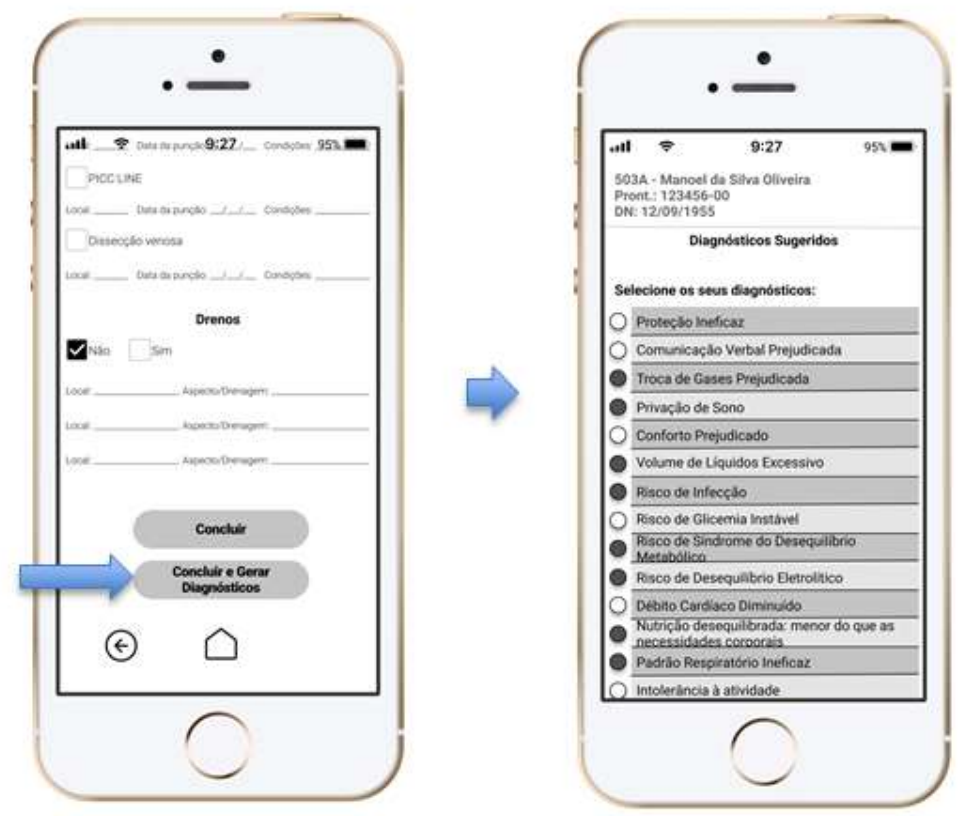

Fonte: Autores.

A Figura 12 demonstra os fatores relacionados que foram identificados pelo registro no Exame Físico e Histórico de Enfermagem. O usuário tem a opção de selecionar o fator que mais contribui para aquele diagnóstico ou mais de um fator.

Figura 11 - Telas de Diagnósticos Sugeridos e tela de Diagnósticos Selecionados. Recife, 2020.
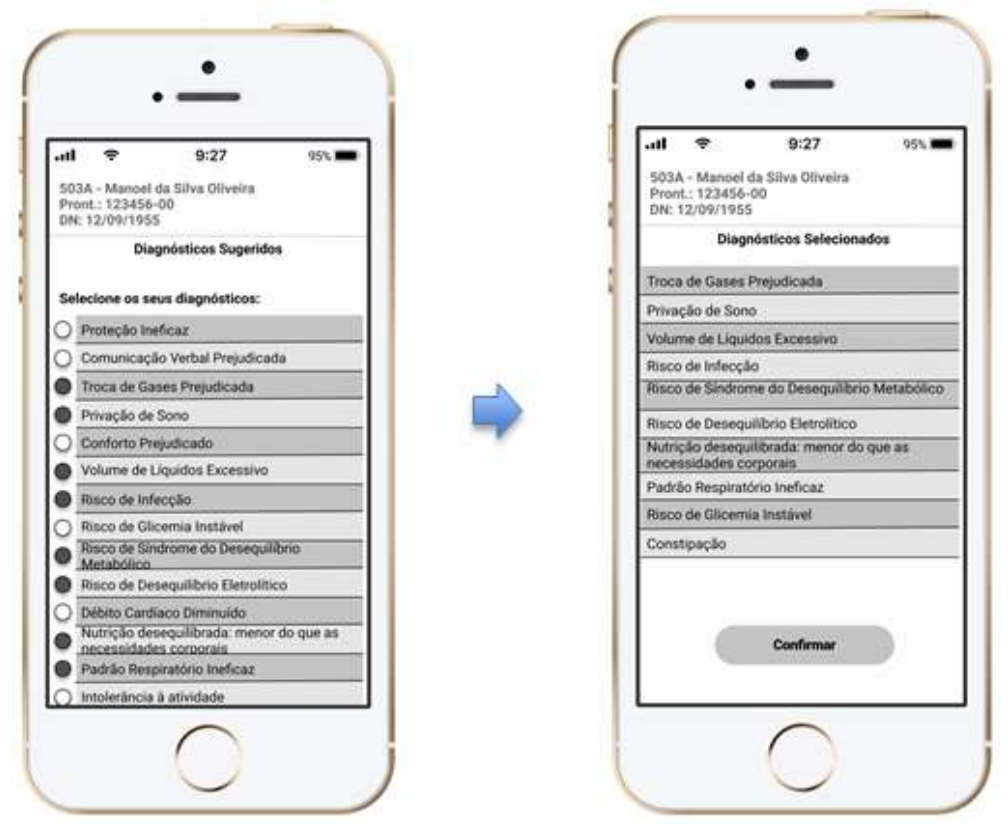

Fonte: Autores. 
Figura 12 - Telas de confirmação dos Diagnósticos Selecionados e tela de Fatores Relacionados. Recife, 2020.
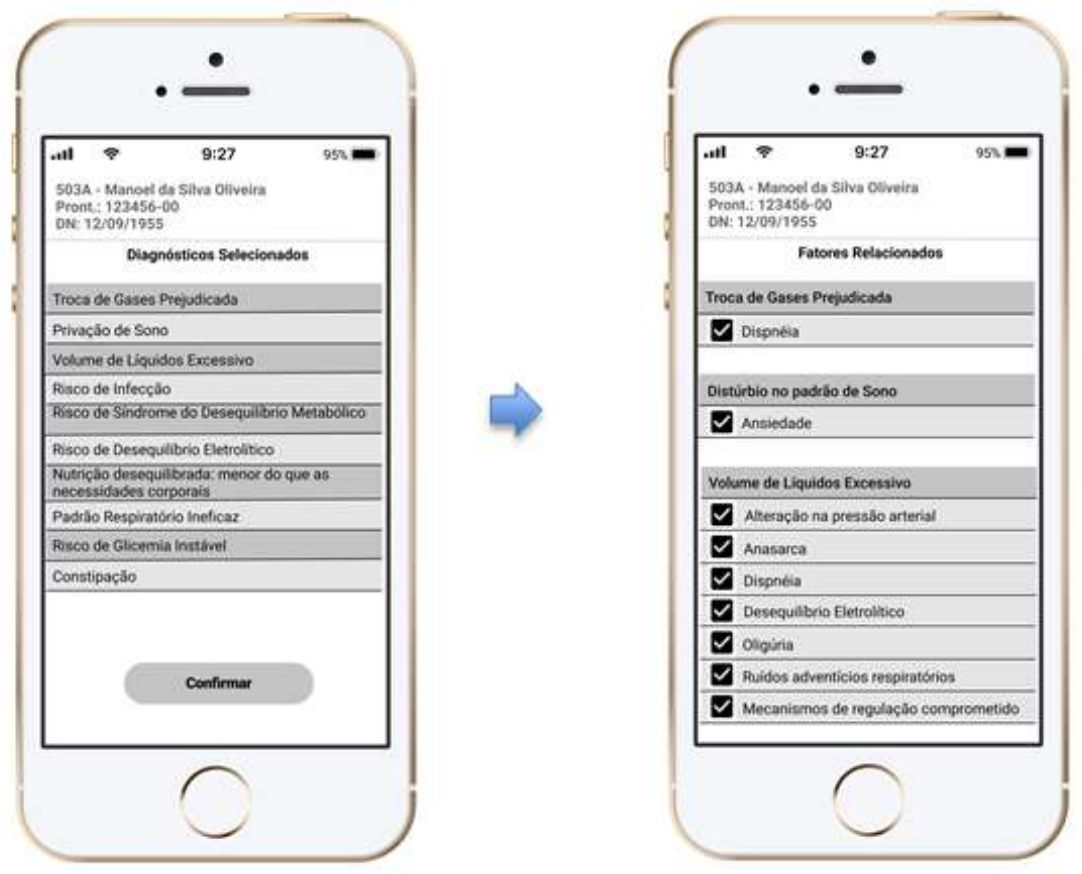

Fonte: Autores.

Após definir os diagnóstico e fatores relacionados, o enfermeiro é encaminhado para a tela de intervenções onde poderá concluir o plano assistencial com uma prescrição de enfermagem individualizada para o paciente. No momento da implementação dos cuidados, o técnico de enfermagem ou o enfermeiro pode checar os horários de forma simples e rápida (Figura 13).

Figura 13 - Telas de Planejamento e Implementação dos cuidados de Enfermagem.
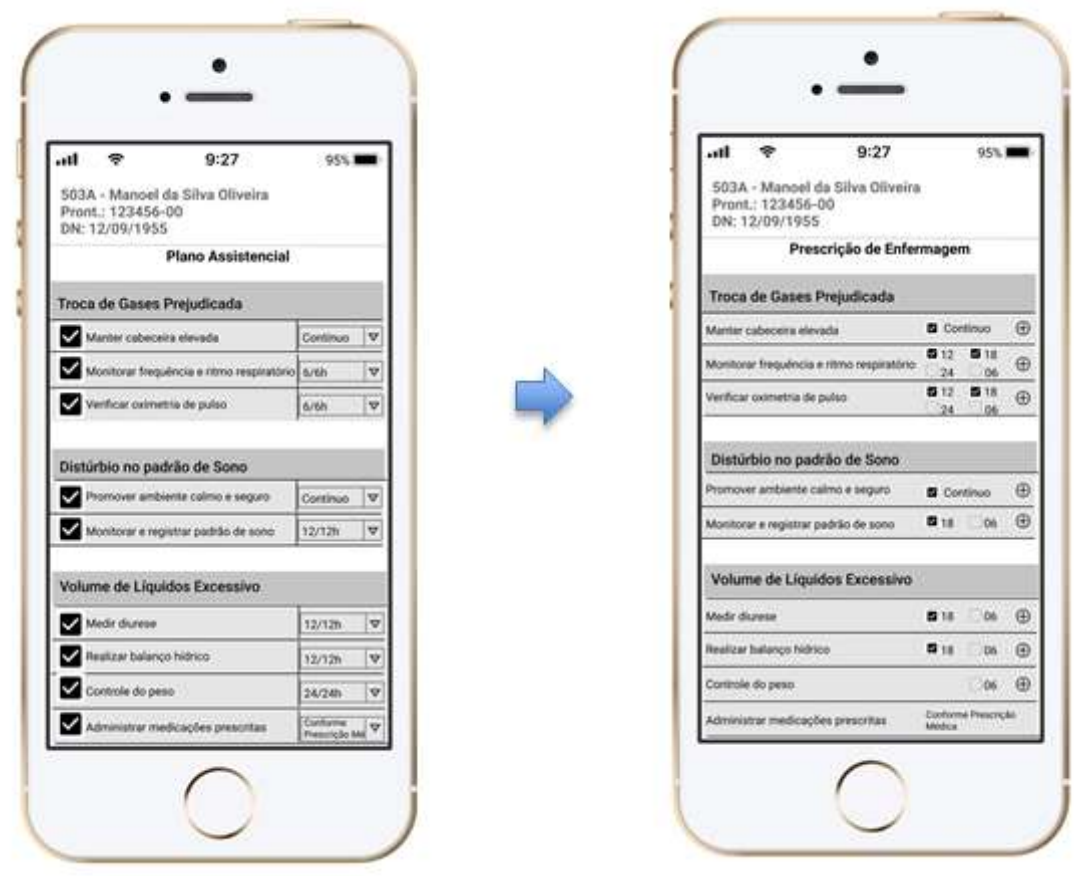

Fonte: Autores. 
Para a realização da Avaliação de Enfermagem o enfermeiro descreve quais diagnósticos foram solucionados, através das intervenções implementadas, utilizando um campo aberto conforme a Figura 14.

O protótipo disponibiliza um campo aberto para que os enfermeiros ou técnicos de enfermagem registrem intercorrências com o paciente ou qualquer evento que não conste na ferramenta (Figura 15).

Figura 14 - Avaliação de Enfermagem. Recife, 2020.

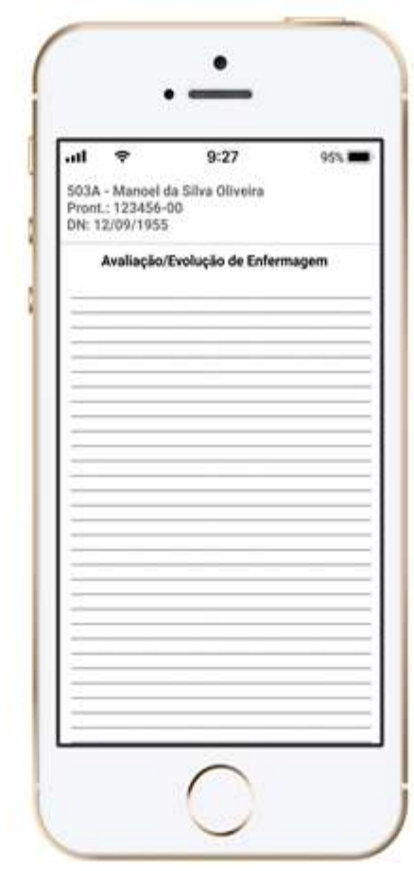

Fonte: Autores.

Figura 15 - Tela que se destina ao registro de Intercorrências/Observações. Recife, 2020.

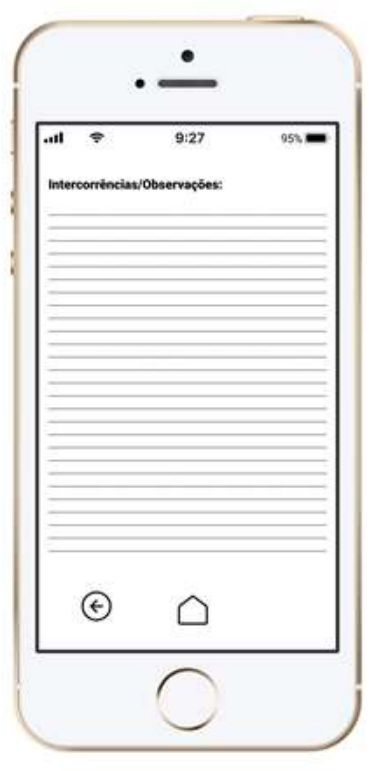

Fonte: Autores. 


\section{Discussão}

O cuidado de enfermagem está conectado com a tecnologia, uma vez que a profissão se embasa em princípios, leis e teorias. As inovações tecnológicas são ações que aprimoram o cuidado, isto é, a tecnologia é fundamental para a organização e sistematização do cuidado de enfermagem. Ao discorrer sobre o cuidado no olhar tecnológico, verificamos a capacidade de inovação do ser humano a fim de melhorar seu cotidiano, dessa forma a tecnologia é a expressão do conhecimento científico de enfermagem transformado para o melhor atendimento (Rocha, et al. 2008).

Uma pesquisa realizada pelo Instituto Brasileiro de Geografia e Estatística (IBGE), constatou que 92,1\% da população brasileira utiliza smartphones como principal fonte de acesso às informações. A principal característica dos dispositivos móveis é a mobilidade, uma vez que podem acompanhar seu usuário 24 horas por dia onde ele estiver (Tibes et al. 2015).

Estudo desenvolvido na cidade de Viçosa/MG em 2017 identificou aumento nas produções sobre o processo de enfermagem (PE) aplicado à utilização de softwares. Em sua maioria as publicações são caracterizadas por estudos descritivos, originados no Brasil e compõe todas as etapas do PE, concluindo assim que o uso de softwares fortalece a prática baseada em evidência e consolida a enfermagem como ciência (Domingos et al. 2017).

No Brasil, diversos pesquisadores vêm trabalhando na construção de tecnologias que qualifiquem o cuidado e promovam segurança ao paciente. Como exemplo pode-se mencionar Martins e Chianca (2016) que elaboraram um software com a finalidade de aplicar de forma fácil e segura o processo de enfermagem a pacientes assistidos em uma unidade de terapia intensiva, usando como base para o protótipo a Teoria das Necessidades Humanas Básicas, da enfermeira Wanda de Aguiar Horta, adotando os diagnósticos de enfermagem da NANDA-Internacional. A escolha da teoria de Horta, através do modelo das necessidades humanas básicas, proporciona ao enfermeiro uma avaliação do ser humano em sua totalidade, garantindo o planejamento da assistência ao visualizar as manifestações das necessidades apresentadas pelo paciente.

A SAE é uma metodologia de organização, planejamento e execução de ações sistematizadas, que são realizadas pela equipe durante o período em que o indivíduo se encontra sob a assistência da enfermagem. A Resolução do Conselho Federal de Enfermagem (COFEN) 272/2002, revogada pela resolução 358/2009 considera a SAE, uma atividade privativa do enfermeiro, que utiliza método e estratégia de trabalho científico para a identificação das situações de saúde/doença (Santos, 2014).

Pesquisa realizada em Sobral/CE teve como objetivo construir um software para a segurança do paciente em pediatria e concluiu que a aplicação desta tecnologia contribui com a qualificação da assistência de enfermagem ao paciente pediátrico oferecendo-lhe mais segurança durante a internação (Araújo et al. 2018).

Um estudo avaliou a realização da Sistematização de Enfermagem em UTI Neonatal através de dispositivos móveis, foram identificadas as dificuldades de manuseio do sistema, reconhecido a importância do protótipo para SAE em dispositivo móvel e sugerido modificações no sistema para adequação à realidade assistencial. Neste, as falas das enfermeiras demonstraram consenso quanto à importância de um aplicativo para SAE em tecnologia móvel. As vantagens mencionadas se referiam a requisitos como mobilidade e agilidade para realizar a evolução do paciente e elaborar o plano de cuidados, otimizando o tempo, além da flexibilidade permitida na gerência das ações de cuidado com o uso do tablet (Rezende, 2016).

Portanto, com a criação do protótipo será possível construir um aplicativo capaz de gerar automaticamente os diagnósticos de enfermagem, assim como seu plano assistencial e os resultados esperados baseados nos dados coletados durante o preenchimento do exame físico do paciente. A utilização de um aplicativo móvel possibilita maior agilidade no fluxo de informações e interação entre os profissionais envolvidos no cuidado, ampliando o tempo de dedicação ao paciente, facilitando assim o desenvolvimento dos diagnósticos, planejamento e intervenções da sistematização da assistência de enfermagem prestada. 


\section{Conclusão}

A partir deste estudo, tornou-se factível, a construção de um protótipo de aplicativo móvel para realização do processo de enfermagem voltado para o paciente renal, este protótipo servirá como instrumento de estudos posteriores, realizados os devidos ajustes para o alcance do resultado desejado. A aplicação de recursos tecnológicos na área da saúde favorece o trabalho do enfermeiro, principalmente por aumentar o tempo disponível para as atividades relacionadas ao cuidado, proporcionando um registro informatizado, individualizado, rápido e eficiente para cada cliente. Além de contribuir e dar continuidade a pesquisas nessa área, a fim de aprimorar os avanços tecnológicos voltados para a sistematização da assistência de enfermagem realizada pelo protótipo.

Sugere-se novas produções científicas acerca da temática em questão, proporcionando assim, reflexões críticas atualizadas. Estes futuros estudos compreendem: consolidar o protótipo como um software de uso efetivo pelas instituições hospitalares como apoio a Sistematização da Assistência de Enfermagem; encaminhamento do protótipo para design e programação e avaliação/validação; patentear o software, objeto do presente trabalho, tão logo esteja efetivamente operacional.

\section{Referências}

Aquino, P. S. et al. (2010). Análise do conceito de tecnologia na enfermagem segundo o método evolucionário. Acta Paulista de Enfermagem, 23(5), 690696. https://www.scielo.br/pdf/ape/v23n5/17.pdf

Araújo, F. A. C. et al. (2018). Elaboração de software para tomada de decisões clínicas em enfermagem na prevenção de quedas em pediatria. CIAIQ2018. https://www.researchgate.net/publication/326305640_ELABORACAO_DE_SOFTWARE_PARA_TOMADA_DE_DECISOES_CLINICAS_EM_ENFERM AGEM_NA_PREVENCAO_DE_QUEDAS_EM_PEDIATRIA

Bordinhão, R. C. \&Almeida, M. A. (2012). Instrumento de coleta de dados para pacientes críticos fundamentado no modelo das necessidades humanas básicas de horta. Revista Gaúcha de Enfermagem, 33(2), 125-131. https://www.scielo.br/pdf/rgenf/v33n2/18.pdf

Conselho Federal de Enfermagem - Cofen. Resolução COFEN-358/2009. http://www.cofen.gov.br/resoluo-cofen-3582009_4384.html

Crestani Filho, V. J. \& Rodrigues, R. A. C. (2013). Progressão da doença renal crônica: experiência ambulatorial em SantarémPará. Brazilian Journal of Nephrology, 35(20, 99-106. https://bjnephrology.org/en/article/progression-of-chronic-kidney-disease-ambulatory-experience-insantarem-para/

Cunha, S. M. B. \& Barros, A. L. B. L. (2005). Análise da implementação da Sistematização da Assistência de Enfermagem, segundo o Modelo Conceitual de Horta. Revista Brasileira de Enfermagem, 58(5), 568-572. https://doi.org/10.1590/S0034-71672005000500013.

Almeida, O. A. E., et al. (2019). Engaging people with chronic kidney disease in their own care an integrative review. Ciência \& Saúde Coletiva, vol. 24, no. 5. Gale Academic OneFile, https://link-gale.ez16.periodicos.capes.gov.br/apps/doc/A593352842/AONE?u=capes\&sid=AONE\&xid=717f532e.

Carvalho, E. C. \& Bachion, M. M. (2009). Processo de enfermagem e sistematização da assistência de enfermagem-intenção de uso por profissionais de enfermagem. Revista Eletrônica de Enfermagem, 11(3). https://doi.org/10.5216/ree.v11.47056

De Oliveira, A. P. C. et al. (2012). Sistematização da assistência de enfermagem: implementação em uma Unidade de Terapia Intensiva. Revista da Rede de Enfermagem do Nordeste, 13(3), 601-612. https://www.redalyc.org/pdf/3240/324027982013.pdf

De Oliveira, A. R. F. \& De Menezes Alencar, M. S. (2017). O uso de aplicativos de saúde para dispositivos móveis como fontes de informação e educação em saúde. RDBCI: Revista Digital de Biblioteconomia e Ciência da Informação, 15(1), 234-245. https://doi.org/10.20396/rdbci.v15i1.8648137

Domingos, C. S. et al. (2017). A aplicação do processo de enfermagem informatizado: revisão integrativa. Enfermería Global, 16(4), 603-652. http://dx.doi.org/10.6018/eglobal.16.4.278061

Dos Santos Barros, W. C. T. et al. (2019). Aplicativo para avaliação do nível de consciência em adultos: produção tecnológica em enfermagem. Cogitare Enfermagem, v. 24. https://revistas.ufpr.br/cogitare/article/view/60338

Dos Santos, W. N. et al. (2014). Sistematização da Assistência de Enfermagem: o contexto histórico, o processo e obstáculos da implantação. JMPHC| Journal of Management \& Primary Health Care. 5(2), 153-158. https://doi.org/10.14295/jmphc.v5i2.210

Fassbinder, T. R. C. et al. (2015). Capacidade funcional e qualidade de vida de pacientes com doença renal crônica pré-dialítica e em hemodiálise-um estudo transversal. J. Bras. Nefrol., 37(1), 47-54. http://dx.doi.org/10.5935/0101-2800.20150008

Fonseca De Oliveira, A. R. \& Alencar, M. S. De M. (2017). O uso de aplicativos de saúde para dispositivos móveis como fontes de informação e educação em saúde. RDBCI: Revista Digital de Biblioteconomia e Ciência da Informação, 15(1), 234-245. https://doi.org/10.20396/rdbci.v15i1.8648137

Fortes, V. L. F. et al. (2013). O itinerário da doença renal crônica: do prenúncio à descoberta. Revista da Rede de Enfermagem do Nordeste, 14(3), 531540. https://www.redalyc.org/pdf/3240/324027991009.pdf 
George, J. B. (1993). Teorias de enfermagem: os fundamentos para a prática profissional. In: Teorias de enfermagem: os fundamentos para a prática profissional. 338-338. https://pesquisa.bvsalud.org/portal/resource/pt/bde-5292

Lima, J. J. de; Vieira, L. G. D. \& Nunes, M. M. (2018). Proceso de enfermería informatizado: construcción de tecnología móvil para uso en neonatos. Revista Brasileira de Enfermagem, 71, 1273-1280. https://www.scielo.br/pdf/reben/v71s3/pt_0034-7167-reben-71-s3-1273.pdf

Lopez, P. S., et al. (2014). Could the type of treatment for chronic kidney disease affect the auditory system?: O tratamento da doença renal crônica pode afetar a audição?. Brazilian Journal of Otorhinolaryngology, 80(1), 54-59. http://www.bjorl.org/pt-o-tratamento-da-doenca-renal-articulo-X2530053914746537

Martins, M. C. T. \& Chianca, T. C. M. (2016). Construção de um software com o com o Processo de Enfermagem em Terapia Intensiva. Journal of Health Informatics, 8(4). http://www.jhi-sbis.saude.ws/ojs-jhi/index.php/jhi-sbis/article/view/420

Merhy, E. E. \& Chakkour, M. (1997). Em busca de ferramentas analisadoras das tecnologias em saúde: a informação e o dia a dia de um serviço, interrogando e gerindo trabalho em saúde. https://digitalrepository.unm.edu/lasm_pt/326/

Moffatt, K. et al. (2003). Participatory designwith aphasic individuals. http://citeseerx.ist.psu.edu/viewdoc/downloa

$\mathrm{d}$ ?doi=10.1.1.83.9901\&rep=rep1\&type=pdf

Morsch, C. \& Veronese, F. J. V. (2011). Doença renal crônica: definição e complicações. Clinical \& Biomedical Research, 31(1). https://seer.ufrgs.br/hcpa/article/view/20014

Neves, R. de S. (2006). Sistematização da assistência de enfermagem em unidade de reabilitação segundo o modelo conceitual de Horta. Revista Brasileira de Enfermagem, 59(4), 556-559. https://doi.org/10.1590/S0034-71672006000400016

Palomares M. L. E. \& Marques I. R. (2010). Contribuições dos sistemas computacionais na implantação da sistematização da assistência de enfermagem. $J$ Health Inform [Internet]. http://www.jhi-sbis.saude.ws/ojs-jhi/ index.php/jhi-sbis/article/view/94/34

Pagliuca, L. M. F. (1993). Os princípios da teoria das necessidades humanas básicas e sua aplicabilidade para o paciente com indicação de transplante de córnea. Revista Brasileira de Enfermagem, 46(1), 21-31. https://doi.org/10.1590/S0034-71671993000100003

Pereira, A. S. et al. (2018). Metodologia da pesquisa científica. UFSM. https://repositorio.ufsm.br/bitstream/handle/1/15824/Lic_Computacao_MetodologiaPesquisa-Cientifica.pdf?sequence $=1$.

Pimpão, F. D. et al. (2010). Percepção da equipe de enfermagem sobre seus registros: buscando a sistematização da assistência de enfermagem. Repositório Institucional da Universidade de Rio Grande do Norte. http://repositorio.furg.br/handle/1/1570

Pissaia, L. F. et al. (2018). Impacto de tecnologias na implementação da sistematização da assistência de enfermagem hospitalar: uma revisão integrativa. Revista de Epidemiologia e Controle de Infecção, 8(1), 92-100. http://dx.doi.org/10.1758/reci.vlil.8953

Polit, D. F.; Beck, C. T. (2011). Fundamentos de pesquisa em enfermagem: avaliação de evidências para a prática da enfermagem. Artmed Editora.

Polit, D. F.; Hungler, B. P. (1995). Fundamentos de pesquisa em enfermagem. In: Fundamentos de pesquisa em enfermagem. p. $391-391$.

Pressman, R.; Maxim, B. (2016). Engenharia de Software. (8a ed.), McGraw Hill Brasil.

Reppetto, M. Â. \& Souza, M. F. de. (2009). Avaliação da realização e do registro da sistematização da assistência de enfermagem (SAE) em um hospital universitário. Revista Brasileira de Enfermagem, 58(3), 325-329. https://doi.org/10.1590/S0034-71672005000300014

Rezende, L. C. M.; Santos, S. R. dos \& Medeiros, A. L. (2016). Avaliação de um protótipo para Sistematização da Assistência de Enfermagem em dispositivo móvel. Rev. Latino-Am. Enfermagem, Ribeirão Preto, 24, e2714. http://www.scielo.br/scielo.php?script=sci_arttext\&pid=S0104-11692016000100343\& $\operatorname{lng}=$ en\&nrm=iso

Rocha, P. K. et al. (2008). Cuidado e tecnologia: aproximações através do Modelo de Cuidado. Revista Brasileira de Enfermagem, 61(1), 113-116, 2008. http: //dx.doi.org/10.17058/reci.v1i1.8953

Sabino, L. M. M. de et al. (2016). Uso de tecnologia leve-dura nas práticas de enfermagem: análise de conceito. http://www.scielo.org.co/scielo.php? pid=S1657-59972016000200010\&script=sci_abstract\&tlng=pt\#: :text=A\%20tecnologia\%20leve\%2Ddura\%2 0ultrapassa,v\%C3\%ADnculo \%20entre\%20pro fission al\% $20 \mathrm{e} \% 20$ cliente.

Santos, Z. M. S. A. (2016). Tecnologia em Saúde-Aspectos teórico-conceituais. Tecnologias em saúde: da abordagem teórica à construção e aplicação no cenário do cuidado. Fortaleza: EdUECE, 12-22. http://www.scielo.org.co/scielo.php?pid=S1657-59972016000200010\&script=sci_abstract\&tlng=pt

Soares, C. R.; Peres, H. H. C. \& De Oliveira, N. B. (2018). Processo de Enfermagem: revisão integrativa sobre as contribuições da informática. Journal of Health Informatics, 10(4). http://www.jhi-sbis.saude.ws/ojs-jhi/index.php/jhi-sbis/article/view/550

Sommerville, I. (2007). Engenharia de Software. (8a ed.), Pearson Education do Brasil.

Souza, J. S. de et al. (2018). Percepção dos profissionais de enfermagem sobre a sistematização da assistência de enfermagem informatizada em um hospital universitário. https://repositorio.ufu.br/bitstream/123456789/23851/1/PercepcaoProfissionaisEnfermagem.pdf

Tibes, C. M. dos S. et al. (2015). Aplicativo móvel para prevenção e classificação de úlceras por pressão. Universidade Federal de São Carlos. https://repositorio.ufscar.br/bitstream/handle/ufscar/3287/6796.pdf?sequence=1

Trepichio, P. B. et al. (2013). Perfil de los pacientes y carga de trabajo de enfermería en la unidad de nefrología. Revista Gaúcha de Enfermagem, 34(2), 133139. https://doi.org/10.1590/S1983-14472013000200017 
Research, Society and Development, v. 10, n. 3, e21810313226, 2021

(CC BY 4.0) | ISSN 2525-3409 | DOI: http://dx.doi.org/10.33448/rsd-v10i3.13226

Zhang, H., et al. (2019). Modular construction for interacting with software. U.S. Patent $\mathrm{n}$. 10,188,939. https://scholar.google.com.tw/citations?user=6 NAxXqsAAAAJ\&hl=zh-TW 